\title{
Perceptual learning improves contrast sensitivity, visual acuity, and foveal crowding in amblyopia
}

\author{
BAROLLO Michele ${ }^{1,2}$, CONTEMORI Giulio ${ }^{1,2}$, BATTAGLINI Luca ${ }^{1,2}$, PAVAN Andrea ${ }^{3}$, and \\ CASCO Clara ${ }^{1,2}$
}

${ }^{1}$ Department of General Psychology, University of Padova, Padova, Italy

${ }^{2}$ Neuro.Vis.U.S. Laboratory, University of Padova, Padova, Italy

${ }^{3}$ University of Lincoln, School of Psychology, Brayford Pool LN6 7TS, United Kingdom

Corresponding author: BAROLLO Michele, phone: +39 0498274648, email: michele.barollo@unipd.it

\begin{abstract}
Background

Amblyopic observers present abnormal spatial interactions between a low-contrast sinusoidal target and high-contrast collinear flankers. It has been demonstrated that perceptual learning (PL) can modulate these low-level lateral interactions, resulting in improved visual acuity and contrast sensitivity.

Objective
\end{abstract}

We measured the extent and duration of generalization effects to various spatial tasks (i.e., visual acuity, Vernier acuity, and foveal crowding) through PL on the target's contrast detection.

Methods

Amblyopic observers were trained on a contrast-detection task for a central target (i.e., a Gabor patch) flanked above and below by two high-contrast Gabor patches. The pre- and post-learning

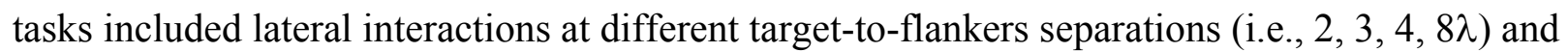
included a range of spatial frequencies and stimulus durations as well as visual acuity, Vernier acuity, contrast-sensitivity function, and foveal crowding.

Results

The results showed that perceptual training reduced the target's contrast-detection thresholds more for the longest target-to-flanker separation (i.e., $8 \lambda$ ). We also found generalization of PL to different stimuli and tasks: contrast sensitivity for both trained and untrained spatial frequencies, visual acuity for Sloan letters, and foveal crowding, and partially for Vernier acuity. Follow-ups after 5-7 
months showed not only complete maintenance of PL effects on visual acuity and contrast sensitivity function but also further improvement in these tasks.

\section{Conclusion}

These results suggest that PL improves facilitatory lateral interactions in amblyopic observers, which usually extend over larger separations than in typical foveal vision. The improvement in these basic visual spatial operations leads to a more efficient capability of performing spatial tasks involving high levels of visual processing, possibly due to the refinement of bottom-up and topdown networks of visual areas.

Keywords

Amblyopia; Perceptual Learning; Visual Acuity; Crowding; Contrast Sensitivity 


\section{Introduction}

Amblyopia is a developmental visual disorder characterized by reduced visual acuity (VA), contrast sensitivity function (CSF), Vernier acuity, and as well as presence of foveal crowding (see Polat, 2009 for a review). These abnormalities cannot be improved by refractive correction of the amblyopic eye and occur in the absence of ocular diseases that could contribute to reduced visual acuity. Anomalous spatial interactions (Bonneh, Sagi, \& Polat, 2007; Levi, Hariharan, \& Klein, 2002; Polat, Bonneh, Ma-Naim, Belkin, \& Sagi, 2005; Polat, Ma-Naim, Belkin, \& Sagi, 2004; Polat, Sagi, \& Norcia, 1997) have also been observed in the amblyopic eye; these are mainly suppressive and extend over larger distances than in normal foveal vision, similar to the peripheral vision of non-amblyopic observers (Levi et al., 2002; Maniglia et al., 2011).

The evidence that perceptual learning (PL), defined as improved performance after repeated practice in a sensory task, is associated with neural plasticity in human adults (see Sagi, 2011, for a review) has led several studies to inquire about the potential of PL as a non-invasive treatment for amblyopia.

Specificity -improvement regarding the trained stimulus that is considered to be encoded by low-level brain mechanisms - vs. generalization are debated aspects of perceptual learning that have become crucial for translational research. Although specificity is desirable because it reflects plasticity in the adult visual system, it is troublesome from the point of view of rehabilitative outcomes because effective rehabilitation should be generalized to visual tasks performed in daily life. This is why several studies have investigated the conditions under which a trained task transfers to some related untrained tasks (Chen, Chen, Fu, Chien, \& Lu, 2008; Chung, Li, \& Levi, 2008; Chung, Li, \& Levi, 2006; Huang, Zhou, \& Lu, 2008; Levi \& Polat, 1996; Levi, 2005; Levi, Polat, \& Hu, 1997; Li \& Levi, 2004; Li, Provost, \& Levi, 2007; Li, Young, Hoenig, \& Levi, 2005; Li, Howard, Parrish, \& Gottfried, 2008; Polat, 2009; Polat et al., 2004; Zhou et al., 2006). Amblyopic patients have been trained through several PL tasks, such as Vernier acuity (Levi \& Polat, 1996; Levi et al., 1997), position discrimination in noise (Li, Klein, \& Levi, 2008; Li et al., 2007, 2005; Li \& Levi, 2004), identification of either contrast-defined or luminance-defined letters (Chung et al., 2008; Chung et al., 2006; Levi, 2005), and contrast detection of sinusoidal stimuli presented either isolated (Huang et al., 2008; Zhou et al., 2006) or together with high-contrast collinear flankers (Campana, Camilleri, Pavan, Veronese, \& Giudice, 2014; Chen et al., 2008; Polat, 2009; Polat et al., 2004). Although PL-dependent improvement in visual acuity has been shown after almost all of these tasks, a benefit for other visual tasks has only been found with some PL procedures but not others. For example, PL on Vernier acuity - a task based on discrimination 
between stimuli - does not transfer to detection (Levi \& Polat, 1996; Levi et al., 1997), and PL on identification of contrast-defined letters does not transfer to luminance-defined letters (Chung et al., 2008). However, PL on contrast detection of sinewave gratings or Gabor patches generalizes to both visual acuity and untrained spatial frequencies (Huang et al., 2008; Polat et al., 2004; Zhou et al., 2006), although transfer to Vernier acuity has not been tested yet.

To be truly effective in improving visual functions, PL should reduce foveal crowding in the amblyopic eye. Indeed, amblyopic participants often exhibit strong crowding effects, i.e., impaired discriminability of target objects when presented in clutter, either assessed directly (Bonneh et al., 2007; Levi et al., 2002; Polat et al., 2005; Polat et al., 1997) or demonstrated by poorer visual acuity when letters are presented in a line than when in isolation (Flom, Weymouth, \& Kahneman, 1963; Giaschi, Regab, Kraft, \& Kothe, 1993; Li \& Levi, 2004; Li et al., 2007; Simmers, Gray, McGraw, \& Winn, 1999). Wong et al. (2005) and Polat et al. (2004) suggested that predominantly inhibitory cortical interactions may account for foveal crowding in the amblyopic eye. Li and Levi (2004) and $\mathrm{Li}$ and colleagues (2007) interpreted greater improvement in discriminating the misalignment between pairs of line segments than in single Snellen letter acuity as PL-dependent reduced crowding. However, a direct measure of the effect of PL in reducing crowding - as demonstrated by the reduced minimum distance between the target and flankers needed to cancel out the detrimental effect of flankers on target recognition (i.e., critical spacing) - has never been provided.

Another critical concern is how PL improves abnormal visual functions in the amblyopic eye. One line of research attempts to distinguish the effect of PL on spatial uncertainty (internal positional noise) from its effect on sampling efficiency (the ability to extract stimulus information) - the two main hypotheses for reduced spatial vision in amblyopia ( Li et al., 2008, 2005; Li \& Levi, 2004). Polat and colleagues $(2004,2009)$ focused on anomalous spatial interactions using a lateral masking paradigm to train amblyopic adults and children to detect Gabor patches presented with collinear high-contrast Gabor flankers. They found that training on increasingly high spatial frequencies significantly improved the contrast sensitivity function (CSF) and visual acuity. These improvements are compatible with a modulation of lateral interactions. Indeed, the initial spatial suppression that amblyopes manifested at intermediate target-to-flanker separations (at which normal-sighted observers present facilitation) was strongly reduced or cancelled out by PL (Polat et al., 2004).

Moreover, although a long-term follow-up is required to determine the actual efficacy of PL as a treatment, few studies have assessed whether the PL-related improvement and its transfer are maintained at follow-up to show that PL is an effective tool for rehabilitation of amblyopia. These 
studies found sustained improvement lasting from 3 to 12 months after the tratment (Li et al., 2013; Liu, Zhang, Jia, Wang, \& Yu, 2011; Polat et al., 2004; To et al., 2011). Interestingly, in Polat et al. (2004), the 12-month follow-up testing showed not only complete retention but also additional improvement in CSF at spatial frequencies of 12 and 18 cycles per degree $\left(\right.$ cycles $\left./{ }^{\circ}\right)$.

In the present study, ten participants with either strabismic amblyopia (binocular misregistration) or anisometropic amblyopia (due to high refractive error), who were aged between 6 and 53 years, participated in a perceptual-learning protocol based on a contrast-detection task with lateral masking. Many of them underwent occlusion treatment that was ineffective, either because it was discontinuous or due to little compliance (Table 1). We used a contrast-detection paradigm because previous studies showed greater improvement, good maintenance, and a high degree of generalization (Levi, 2012). The training sessions consisted of measuring contrast thresholds for detecting, in the amblyopic eye, small and foveally presented Gabor patches, displayed along with collinear flankers with the same spatial frequency but higher contrast than the central target. For all of the trained observers, we assessed whether the PL-dependent improvement varied with spatial frequency and target-to-flanker separations, expressed in multiples of the carrier's wavelength $(\lambda)$ of the Gabor patch. Transfer of PL was assessed in visual acuity of isolated letter recognition, visual hyperacuity, CSF, and foveal crowding. Differently from previous studies, we did not test CSF with a standard contrast sensitivity chart or use the same contrast-detection task employed for the training (Huang et al., 2008; Zhou et al., 2006). Instead, we measured CSF with an orientationdiscrimination task to assess whether PL based on contrast detection enhanced the CSF, as measured with a different task. Moreover, previous studies have not directly assessed whether PL on contrast-detection tasks with lateral masking stimuli is suitable for reducing critical spacing in an untrained foveal crowding task. Maniglia and coworkers (2011) showed that after PL in a contrastdetection task with a lateral masking paradigm, peripheral crowding was strongly reduced in both normal-sighted observers and patients with macular degeneration (Maniglia et al., 2016). Since anomalous foveal interactions are thought to be similar to those in the peripheral vision of nonamblyopic observers, we asked whether PL in contrast detection of lateral masking may reduce foveal crowding in the amblyopic eye.

Additionally, visual acuity (for 7 participants) and CSF (for 6 participants) were tested 5 to 7 months after the training. We aimed not only to replicate the result that PL transfer effects were retained but also to determine whether, in the absence of further training, there was an additional improvement in visual acuity and CSF at high spatial frequencies, as previously reported by Polat et 
al. (2004). In fact, adding evidence on this topic would provide some insight on the involvement of long-term neural plasticity in PL (Levi, 2012).

\section{Methods}

\subsection{Participants}

Ten amblyopic and ten age-matched non-amblyopic observers participated in the study. The amblyopic participants provided ophthalmological information before testing, including full refractive correction, a detailed ophthalmic history, diagnosis of amblyopia, and subjective refraction with best visual acuity (VA). To join the study, the patients must have had stable visual acuity and subjective refraction in both their amblyopic and non-amblyopic eyes for at least six months. The difference in acuity between the two eyes was $>0.2 \log$ MAR for the monocular amblyopes, except for one subject, whose difference was $0.12 \log$ MAR. Seven participants underwent occlusion therapy, which either failed or was ineffectively concluded because of poor compliance. Table 1 reports the clinical details for each participant. Two participants did not fully meet the inclusion criteria (absence of ocular diseases that could contribute to reduced visual acuity and learning difficulties): according to their ophthalmological reports, $\mathrm{P} 1$ had recovered from a corneal ulcer three years before the testing, and P9 was diagnosed with ametropic amblyopia (high hypermetropia with astigmatism) associated with albinism and nystagmus. One participant, P4, had a borderline score on a sustained attention assessment, although the participant's reduced attentional capacity did not compromise task performance.

Single letter monocular visual acuity (Table 1) was assessed before the PL sessions began, and corrective lens were not changed during the training. All of the participants were compliant during the training, although two of the children (P1 and P6) sometimes needed to be spurred by their parents. The research adhered to the tenets of the Declaration of Helsinki and was conducted under the experimental protocol for human subjects. Furthermore, it was approved by the Ethics Committee of the School of Psychology of the University of Padova (protocol no. 1931). Written informed consent was obtained from each participant or their parents after detailed explanation of the study.

[Table 1 about here]

\subsection{Stimuli}




\subsubsection{PL stimuli}

PL stimuli were generated using Matlab Psychtoolbox (Brainard, 1997; Pelli, 1997) and displayed on a LCD monitor with a refresh rate of $60 \mathrm{~Hz}$ and 8 bits of luminance resolution. The choice to use this basic hardware was made to accommodate participants through user-friendly home-based training, and it is justified by the high contrast thresholds of the participants in the amblyopic eye. In fact, the contrast thresholds in each case reached the limits of the hardware in use (i.e., 0.01 Michelson contrast). Stimuli were generated using a gamma-corrected lookup table (LUT) to ensure display linearity for the LCD monitors used at each participant's home or at the clinical centre. The gamma correction for each colour channel was applied through a LCD monitorspecific calibration with the Spyder 4 Elite colorimeter (DataColor, Lawrenceville, NJ, USA); the mean luminance was $50 \mathrm{~cd} / \mathrm{m}^{2}$. The stimuli software was set up for the screen resolution to ensure the correct representation of spatial frequencies at the viewing distance of $57 \mathrm{~cm}$.

The stimuli were Gabor patches consisting of a cosinusoidal carrier enveloped by a stationary Gaussian. Each Gabor patch was characterized by its wavelength $(\lambda)$, its phase $(\phi)$, and the standard deviation $(\sigma)$ of the luminance Gaussian envelope in the (x,y) space of the image. Formally, each Gabor patch can be expressed as follows:

$$
G(x, y)=\cos \left(\frac{2 \pi}{\lambda} x+\varphi\right) e^{\left(-\frac{x^{2}+y^{2}}{\sigma^{2}}\right)}
$$

with $\sigma=\lambda$ and $\phi=0$ (even symmetric). The Gabors' sensitivity function (SF) used for the PL session was within the range of 3 to 12 cycles $/{ }^{\circ}$. The configuration used consisted of a centrally presented Gabor target flanked above and below by two collinear high-contrast Gabor patches (0.6 Michelson contrast) oriented as the target (Campana et al., 2014; Chen et al., 2008; Polat, 2009; Polat et al., 2004). Four configurations of collinear Gabors were trained, each with different global orientations (horizontal, vertical, diagonal $135^{\circ}$ and diagonal $45^{\circ}$ ). Flankers were located at various distances from the target: $2 \lambda, 3 \lambda, 4 \lambda$, and $8 \lambda$, corresponding to $0.67,1,1.33$ and 2.67 deg for 3 cycles $/{ }^{\circ}$ and $0.17,0.25,0.33$ and $0.67 \mathrm{deg}$ for 12 cycles $^{\circ}{ }^{\circ}$ (Figure 1$)$.

[Figure 1 about here]

\subsubsection{Transfer stimuli}




\subsubsection{Visual acuity stimuli}

We used the FrACT (Freiburg Visual Acuity and Contrast Test) software (Bach, 1996) to measure the visual acuity of single letters, as derived from the measurement of the threshold font size of 10 Sloan letters: C, D, H, K, N, O, R, S, V, and Z. In response, the appropriate letter is typed by the experimenter. Any non-Sloan letter was ignored. Observers viewed the letters monocularly for a maximum of $30 \mathrm{~s}$ at a viewing distance of $200 \mathrm{~cm}$. Acoustic feedback was present for both correct and incorrect answers.

\subsubsection{Visual acuity and foveal crowding stimuli}

Thresholds for central visual acuity (eccentric VA) and for the critical space of crowding i.e., the inter-letter distance at which target (i.e., the central letter) identification was unaffected by the presence of flankers - were measured before and after the training period. Stimuli were generated using Matlab Psychtoolbox (Brainard, 1997; Pelli, 1997) and presented at $57 \mathrm{~cm}$. The stimuli were the same 10 white Sloan letters used for the visual acuity test, randomly presented for $133 \mathrm{~ms}$ on a black background. The size of the letters for acuity and the letter-to-letter distance for crowding varied according to an adaptive procedure - the maximum likelihood procedure (Grassi \& Soranzo, 2009; Green, 1990, 1993) - that tracked 79\% of correct responses. Participants had to verbally report the target letter, and the experimenter registered the answer. Each session was terminated after 60 trials.

For foveal crowding, a central target letter was flanked by two different random letters. The size of both the target and flanking letters was set 30\% higher than the VA threshold to have correct identification at the largest target-to-flankers separation. The initial font size for acuity was 40 (streak width of arcmin), while the initial distance between the letters for crowding was set at 3 degrees. In both tasks, an acoustic cue was presented before the stimulus presentation to reduce temporal uncertainty. No feedback was provided.

\subsubsection{CSF stimuli}

CSF was measured using the FrACT software package. The stimuli were Gabor patches of 2 deg (full width at half maximum) with four different orientations (horizontal, vertical, and diagonal at $45^{\circ}$ and $135^{\circ}$ ). The observers performed a four-alternative forced choice (4AFC) orientation discrimination task monocularly. Each stimulus disappeared immediately after the observer's response. The stimuli were displayed for a maximum of $30 \mathrm{~s}$. The contrast of the stimulus was varied according to a BEST PEST procedure and by using FrACT's built-in bit-stealing technique 
to extend the luminance resolution at 10 bits (Tyler, 1997). The viewing distance was $200 \mathrm{~cm}$, and acoustic feedback was provided for incorrect trials. The spatial frequencies tested were 1, 3, 5, 7, 11 and 15 cycles $/{ }^{\circ}$.

\subsubsection{Vernier acuity}

Vernier acuity was measured using FrACT. The stimuli were two line segments (15 x 1.4 arcmin) with a vertical separation between them of 0.5 arcmin. The observers' task was to discriminate with their amblyopic eye (AE) whether the upper line was displaced to the left or right with respect to the lower line. The stimuli were displayed for a maximum of $30 \mathrm{~s}$. The viewing distance was $200 \mathrm{~cm}$, and acoustic feedback was provided for incorrect trials.

\subsection{Procedure}

\subsubsection{Pre- and post-training evaluation}

The pre-training and post-training measurements of visual acuity, CSF, Vernier acuity, and foveal crowding were obtained in the same ways with the same apparatuses and under the same viewing conditions (best optical correction) at the clinical centre. The evaluation of VA was done for all amblyopic eyes (AEs) and non-amblyopic eyes (NAEs). Vernier acuity was measured for the AE of six observers, foveal crowding was measured for the AE of seven participants, and CSF was measured for the AE of all of the observers. The order of testing was counterbalanced. For most of the participants, VA and CSF were also measured once or twice during the training. Moreover, visual acuity (for 7 participants) and CSF (for 6 participants) were tested 5-7 months after the training was terminated.

\subsubsection{PL procedure}

After completing pre-training testing, the LCD monitor calibration with the Spyder 4 Elite colorimeter was applied to the computer used for the training, and the training conditions were set based on the pre-training evaluation. Except for P9, the PL paradigm involved supervised patching of the fellow eye. Note that the daily occlusion duration (30-45 min) was insufficient to explain the effects of PL as being due to patching the fellow eye, even in patients with mild amblyopia (Pediatric Eye Disease Investigator Group, 2003). Multicentre-based training was administrated under supervision of the leading university centre. All of the training sessions were performed either at the clinical centre (for five participants: P2, P3, P7, P8, and P9) or at the clinical centre for 
the first week and at the participants' home afterwards (for five participants: P4, P6, P1, P5, and P10).

The contrast threshold of the target was varied according to a 1-up/3-down staircase (Levitt, 1971). The participants performed a temporal-2AFC. The target was presented in one of the two temporal intervals, whereas the flankers were always presented in both temporal intervals. A fixation mark $(0.18 \mathrm{deg})$ indicated the location of the target on the blank screen before and after each interval.

The observers had to report the temporal interval at which the target was presented. Acoustic feedback was provided for incorrect trials. Each block was terminated after 120 trials or 16 reversals. The contrast thresholds were estimated by averaging the contrast values corresponding to the last 8 reversals. During the training, the target-to-flankers separation was randomly varied within a daily session, whereas the global orientation of the stimulus configuration was varied across four daily sessions in the following sequence: horizontal, diagonal $45^{\circ}$, vertical, and diagonal $135^{\circ}$. The stimulus duration varied from 133 to $500 \mathrm{~ms}$ as reported in Table 2 according to each subject's performance. The participants completed between 21 and 93 sessions in 16-43 weeks. The Gabors' spatial frequency used for the PL session was chosen within the range of 3 to 12 cycles ${ }^{\circ}$. The initial spatial frequency that was trained was set as the highest spatial frequency for which the contrast threshold could be measured in the pretest CSF with FRACT. The next spatial frequency was changed to a higher value, either according to the improvement obtained in the contrastsensitivity function at the intermediate evaluation or according to PL performance (Polat, 2009), when observers consistently obtained a 2-fold threshold reduction (or more) in the conditions of the highest $(8 \lambda)$ and lowest $(2 \lambda)$ target-to flanker separation.

[Table 2 about here]

\section{Results}

\subsection{PL effect on trained task}

Figure 2 shows the results of PL expressed as contrast threshold (Figure 2A, B) and PL enhancement (i.e., threshold modulation; Figure 2C, D) as a function of the target-to-flankers separations (TtFS), for low $\left(3\right.$ cycles $^{\circ}, N=7$ ) and high spatial frequencies $\left(\geq 6\right.$ cycles $\left./{ }^{\circ}, N=7\right)$. We performed a two-way repeated-measures ANOVA separately for low and high spatial frequencies, including as factors PL (pre- vs. post-training sessions) and $\operatorname{TtFS}(2 \lambda, 3 \lambda, 4 \lambda$ and $8 \lambda)$. The results showed that PL reduces contrast thresholds at both low $\left(F_{1,6}=8.5, p=0.027\right.$, partial- $\eta^{2}$ 
$=0.59)$ and high spatial frequencies $\left(F_{1,7}=6.2, p=0.041\right.$, partial $\left.-\eta^{2}=0.47\right)$. The effect of TtFS is never significant and the interaction PL $\mathrm{x}$ TtFS is significant only for the low spatial frequency $\left(F_{3,18}=3.5, p=0.037\right.$, partial $\left.-\eta^{2}=0.37\right)$. Bonferroni corrected post hoc reported lower contrast threshold at $8 \lambda$ than at $3 \lambda(p<0.04)$ and $2 \lambda(p<0.047)$.

The reduction of thresholds by PL is confirmed by the negative PL modulation illustrated in Figures 2C and D. A one-way repeated-measures ANOVA on PL modulation showed that the effect of TtFS was only significant for the low spatial frequency $\left(F_{3,18}=7.7, p=0.02\right.$, partial $-\eta^{2}=0.56$; high spatial frequency: $F_{3,21}=0.98, p=0.42$, partial $-\eta^{2}=0.13$ ). Bonferroni corrected post hoc comparisons between TtFS revealed that the PL modulation (negative values) was generally stronger at $8 \lambda$ than at the others TtFS: $8 \lambda$ vs $4 \lambda(p=0.033), 8 \lambda$ vs $3 \lambda(p=0.069)$ and $8 \lambda$ vs $2 \lambda,(p=$ $0.1)$.

[Figure 2 about here]

\subsection{Transfer to visual functions}

Table 3 summarizes individual transfer data for SLOAN acuity, CSF, Vernier acuity, and foveal crowding before PL, after PL, and at the follow-up. Test-retest data for an age-matched control group were also included in Table 3. It should be noticed that for one amblyopic participant (P1), PL did not affect either acuity or CSF. Although, according to the ophthalmological report, she had recovered from corneal ulcer three years before testing, the small corneal leucoma may have justified the inefficacy of PL in improving VA and CSF. Moreover, for one participant (P9) who had bilateral amblyopia from nystagmus, we reported data obtained in binocular vision that were similar to those obtained in monocular testing.

Overall, visual acuity was substantially better after than before PL, for both trained amblyopic eyes (AEs) and for the five tested untrained non-amblyopic eyes (NAEs). Additionally, for the AEs, visual acuity was higher at the follow-up than after PL. Contrast sensitivity for trained SF range (3-7 cycles ${ }^{\circ}$ ) was higher in the trained AEs after PL than before PL and higher at the follow-up than after PL. Contrast sensitivity for untrained low SF was not increased either after PL or at the follow-up with respect to the baseline. Conversely, contrast sensitivity for untrained high spatial frequencies (11 and 15 cycles/ $^{\circ}$ ) was higher after than before PL. VA and CW in the trained AEs were reduced by PL.

[Table 3 about here] 


\subsubsection{Transfer to $V A$}

Figure 3 shows individual and mean SLOAN visual acuity, obtained before and after PL in the amblyopic eye (Figure 3A) and fellow-eye (Figure 3B). Follow-up data were also collected for the amblyopic eye after three months for P6 and after at least six months for P1, P3, P4, P5, P7, P9, and $\mathrm{P} 10$.

One-sample $t$-tests showed a significant higher visual acuity in the amblyopic eye after PL than before PL (difference: $0.18 \log \mathrm{MAR} ;(9)=3.8, p=0.004$ ) and at the follow-up with respect to the performance after PL (difference: $0.098 \log \mathrm{MAR} ; t(7)=2.7, p=0.03$ ). The improvement was also significant when comparing before and after PL in the fellow eye (difference: 0.13 logMAR; $t(7)=2.4, p=0.049)$. Moreover, neither before $(\mathrm{t}(8)=0.042, p=0.97)$ nor after PL $(\mathrm{t}(8)=0.57, p=$ $0.58)$, VA differed between juvenile and adult amblyopia.

[Figure 3 about here]

\subsubsection{Transfer to CSF}

Figure 4A shows the mean normalized CSF measured before and after PL for all participants and at the follow-up for 6 participants. For each participant, CSFs were normalized for the maximum value (i.e., by scaling the contrast sensitivity value with respect to the maximum), which was set to the value of 1 . Contrast sensitivity enhancement after PL expressed as $\log \left(\mathrm{CS}_{\text {post }}\right)-\log \left(\mathrm{CS}_{\text {pre }}\right)$, and at follow-up $\log \left(\mathrm{CS}_{\mathrm{f}-\mathrm{up}}\right)-\log \left(\mathrm{CS}_{\text {post }}\right)$ is also shown in Figure 4B. Results indicate that CSF, measured with an orientation discrimination task (see the Methods section), was enhanced by PL on contrast detection task at medium and high spatial frequencies, but not at low spatial frequencies. Moreover, CSF function at follow-up showed further improvement at medium and high spatial frequencies.

Contrast sensitivity enhancement was evaluated for each spatial frequency, with one-sample twotailed $t$-tests. Results revealed a significant enhancement of contrast sensitivity at intermediate spatial frequencies, both after PL $\left(1\right.$ cycles ${ }^{\circ}: t(8)=-0.34, p=0.17 ; 3$ cycles $/{ }^{\circ}: t(9)=1.3, p=0.22$; 5 cycles $^{\circ}{ }^{\circ}: t(9)=2.1, p=0.069 ; 7$ cycles $/{ }^{\circ}: t(8)=3.2, p=0.012 ; 11$ cycles $/{ }^{\circ}: t(6)=2.63, p=0.039$; 15 cycles $\left.^{\circ}: t(4)=1.5, p=0.21\right)$ and at the follow-up with respect to the post-test $\left(1 \mathrm{cycles} /{ }^{\circ}: t(4)=\right.$ $0.51, p=0.64 ; 3$ cycles $/{ }^{\circ}: t(5)=-0.55, p=0.61 ; 5$ cycles $/{ }^{\circ}: t(5)=2.33, p=0.067 ; 7$ cycles $/{ }^{\circ}: t(5)=$ $3.5, p=0.017 ; 11$ cycles $\left./^{\circ}: t(3)=2.1, p=0.12\right)$. The average contrast sensitivity enhancement did not differ amongst juvenile and adult amblyopic patients $(\mathrm{t}(8)=1.15, p=0.28)$. 
[Figure 4 about here]

\subsubsection{Transfer to Vernier acuity}

The transfer of PL to Vernier acuity is shown in Figure 5, which compares individual and average Vernier acuity obtained before and after PL. Four out of six participants showed increased Vernier acuity following PL. A paired sample $t$-test conducted on the six observers did not reach significance $(t(5)=2.44, p=0.058)$.

[Figure 5 about here]

\subsubsection{Transfer to foveal crowding}

The transfer of PL to foveal crowding is shown in Figure 6, which compares center-center critical spacing (expressed in stroke-units) before and after-PL for each of the seven tested participants and at follow-up for three participants. All tested participants, except P9, showed reduction of crowding following PL on contrast detection with lateral masking configurations, and the difference in critical spacing before and after PL was significant $(t(7)=2.64, p=0.039)$.

[Figure 6 about here]

\section{Discussion}

Our results indicate a general PL-dependent reduction of contrast threshold that was stronger at the largest target-to-flanker separation for the low spatial frequency. Besides, PL transferred to untrained tasks, such as orientation discrimination of both trained and untrained spatial frequencies, visual acuity with Sloan letters, Vernier acuity, and foveal crowding. More importantly, follow-up after 5 to 7 months showed not only an almost complete retention of PL effects, but also further improvement in visual acuity and contrast sensitivity with respect to observers' performances immediately after the training.

\subsection{The effect of PL on lateral interactions}

To our knowledge, only one study addressed the question of whether PL with a lateral masking paradigm modulates lateral interactions in the amblyopic eye (Polat et al., 2004). These 
authors showed, before training, inhibition with $\mathrm{TtFS}<2 \lambda$ and no modulatory effect by the flankers at TtFS $>$ than $6 \lambda$. Moreover, they showed that PL modulates foveal inhibitory spatial interactions in the amblyopic eye. In particular, for a Gabor target of 6 cycles $/{ }^{\circ}$, they found reduced inhibition exerted by flankers placed at a distance of $3 \lambda$ (Bonneh et al., 2007; Levi et al., 2002; Polat et al., 2005; Polat et al., 2004, 1997). Considering that we did not measure contrast thresholds for central Gabors either isolated or flanked by orthogonal Gabors, we cannot make a direct comparison with previous results. However, our results show that, at low spatial frequencies, PL leads to lower contrast detection thresholds for $8 \lambda$ than $2 \lambda$ or $3 \lambda$; therefore, a larger threshold modulation was observed for the largest TtFS. These results may be taken as indirect evidence of PL modulation of long-range lateral interaction in the amblyopic eye. Since the initial spatial frequency trained was 3 cycles $/{ }^{\circ}$ for most participants, it may be that $\mathrm{PL}$ affected lateral interactions more at the beginning of training. A more parsimonious explanation is one which considers the effect of TtFS before PL. Our results differ from those of Polat et al. (2005) and Levi et al. (2002), who found facilitation at 3 cycles $/{ }^{\circ}$, and instead are consistent with those of Polat and Norcia (1997), who found less facilitation at 3 cycles $/{ }^{\circ}$ in particular for collinear configurations. In fact, the results of Polat and Norcia (1997) and our results showed that contrast thresholds do not depend on TtFS before PL. Under the assumption that the effect of TtFS depends on the interplay between two mechanisms (Polat, Mizobe, Pettet, Kasamatsu, \& Norcia, 1998)—one responsible for inhibition, relying on suppressive effects within one receptive field, and the other responsible for facilitation, relying on long-range lateral interactions - the higher PL modulation at the largest TtFS suggests that, at low spatial frequency, PL results in more efficient long-range facilitation of lateral interactions.

\subsection{Transfer of PL to other visual tasks}

Our results confirm previous findings that PL on contrast detection tasks transfers to visual acuity and CSF. The new finding is the transfer of PL to foveal crowding, a phenomenon often reported in amblyopic patients (Bonneh et al., 2007; Levi et al., 2002; Polat et al., 2004, 2005, 1997). On the other hand, there was little transfer to Vernier acuity. The improvement in visual acuity was also found in the untrained fellow-eyes, consistently with previous results ( Levi \& Polat, 1996; Levi et al., 1997; Li et al., 2008; Zhou et al., 2006).

Though the clinical relevance of transfer of PL to untrained tasks, previous studies did not always find that PL transfers across tasks. For example, Levi et al. (1996, 1997) found that PL on detecting the lateral displacement of two high contrast lines (Vernier acuity task) did not transfer to 
contrast detection of one of these lines. Other studies showed that PL to recognize contrast defined letters did not transfer to either luminance-defined letters (Chung et al., 2006) or visual acuity (Chung et al., 2008). However, with exception of Vernier acuity in which the transfer was weak, we found that PL generalizes to different tasks and stimuli. In fact, we found a transfer to visual acuity and CSF tested with a different task than the one used during PL (i.e., 4AFC orientation discrimination vs. 2AFC contrast detection). Additionally, we found that PL transferred to foveal crowding and substantially reduced the critical spacing for crowding. However, although overall significant, the transfer effect is not ubiquitous: only participants having high crowding before PL (P1, P4, P8, and P10) have high transfer effect on this task. Moreover, the level of initial crowding did not depend on either initial acuity or on the type of amblyopia; indeed, different from previous studies (Bonneh, Sagi, \& Polat, 2004; Polat, 2005), strabismic amblyopic were not more likely to show crowding. It should be noted that — different from Hussain et al. (2012) — in our paradigm the reduction of crowding was not a direct consequence of training crowded letters. Instead, it occurred across tasks, since it was a generalization effect of practicing on contrast detection with lateral masking configurations. These across-task transfer effects cannot be explained by task-learning and, indeed, test-retest data for aged matched control group (Table 3) did not show no-task learning.

These transfer effects point to a common mechanism (or mechanisms) that, when strengthen by PL and rendered more efficient, exert their beneficial influence on untrained tasks and stimuli. The result whereby the improvement in visual acuity following PL was not specific for the eye suggests that this mechanism acts beyond the modulatory effect of anomalous lateral interactions in the cortical representation of the amblyopic eye. Based on the assumption that vision is attained by intra-cortical (within visual areas) and inter-cortical (between visual areas) connections (Lamme, Supè, \& Spekreijse, 1998; Robol, Grassi, \& Casco, 2013), it can be hypothesized that improved low level contextual influences, mediated by inter-cortical connections, allow integration of visual responses into a coherent representation via the recurrent intra-cortical processing (both feedforward and feedback). Recurrent intra-cortical processing may translate the low-level improvement of lateral interactions into a better capability of segmenting and binding together incoming local sensory signals into meaningful perceptual structures. Recurrent processing may allow the PL-dependent refinement of lateral interactions at early cortical level to result, at higher stage of visual processing, in more efficient binding operations. In this case, letters identification would be improved both when they are presented as isolated or flanked by other letters. Indeed, it has been shown that the same binding process may underlie feature integration in letter identification and crowding: whereas binding within-target elements subserves contour integration, 
binding together target and distractor elements disrupts contour integration (Chakravarthi \& Pelli, 2011). In addition, inter-cortical recurrent processing allowing feedback from higher to lower processing levels may mediate a more efficient top-down and attention-driven modulation of lowlevel high-resolution tasks, such as Vernier acuity and orientation discrimination of high spatial frequency gratings. The follow-up data discussed below also point to a PL effect not limited to the improvement of low level visual processing but extended to top-down driven improvement in combining these signals into salient percepts.

\subsection{Follow-up}

We found that visual acuity and contrast sensitivity for the trained spatial frequencies were further increased at follow-up several months after the training. This result indicates that not all the learning in the amblyopic eye is concurrent with practice. Previous studies have shown a latent phase lasting several hours during which the ability to perform a visual task further improves in the absence of concomitant training (Gais, Plihal, Wagner, \& Born, 2000; Karni \& Sagi, 1993; Stickgold, Whidbee, Schirmer, Patel, \& Hobson, 2000; Walker, Stickgold, Jolesz, \& Yoo, 2005). However, our transfer tests were performed several months after the training and it is unlikely that overnight consolidation of learning may be the explanation of the further improvement in visual acuity and CSF found at the follow-up with respect to the post-training evaluation. Four patients (P1, P5, P7, and P8) had new glasses after post-test, at least three months before follow-up, but the follow-up data were collected for two of them (P5 had full evaluation and P7 had only VA measured) and these data may have been affected at least partially by the new correction. We speculate that the observed improvement at the follow-up may instead reflect a more efficient recurrent inter- and intra-cortical processing. Follow-up improvement may reflect long-term consolidation of the positive influence of the low-level mechanisms being trained on higher level spatial processing. Moreover, it could, in turn, account for top-down modulation of the central lowlevel visual processing. From our data it could be suggested that recurrent processing in vison may be adaptive in nature as suggested by the result that the improvement at follow-up occurs for the tasks more used in daily life (i.e., visual acuity and contrast sensitivity).

\section{Conclusions}

Amblyopia is a disorder of spatial vision which can be reduced by refining the lateral interconnections between neurons in the primary visual cortex through perceptual learning. Perceptual learning can improve low-level spatial vision modulating facilitatory and inhibitory 
lateral interactions between spatial filters in the primary visual cortex (Gilbert \& Wiesel, 1989). Additionally, we showed that training protocols based on lateral interactions are not stimulus and task specific, but they generalize to a variety of more complex spatial tasks (e.g., contrast sensitivity function, foveal, and peripheral crowding). This suggest that improved lateral interactions by PL may trigger complex cortico-cortical feedforward and feedback processing (Lamme et al., 1998; Robol et al., 2013), thus improving perceptual grouping and segmentation of forms at higher cortical levels, so to achieve salient perception of visual forms.

\section{Acknowledgements}

This research was supported by the University of Padova (Italy, authors MB, GC, LB, and CC) and the University of Lincoln (UK, author AP).

\section{References}

Bach, M. (1996). The Freiburg Visual Acuity Test-Automatic Measurement of Visual Acuity. Optometry and Vision Science : Official Publication of the American Academy of Optometry, 73(1), 49-53. https://doi.org/10.1097/00006324-199601000-00008

Bonneh, Y. S., Sagi, D., \& Polat, U. (2004). Local and non-local deficits in amblyopia: acuity and spatial interactions. Vision research, 44(27), 3099-3110.

Bonneh, Y. S., Sagi, D., \& Polat, U. (2007). Spatial and temporal crowding in amblyopia. Vision Research, 47(14), 1950-1962. https://doi.org/10.1016/j.visres.2007.02.015

Brainard, D. H. (1997). The Psychophysics Toolbox. Spatial Vision, 10(4), 433-436. https://doi.org/10.1163/156856897X00357

Campana, G., Camilleri, R., Pavan, A., Veronese, A., \& Giudice, G. Lo. (2014). Improving visual functions in adult amblyopia with combined perceptual training and transcranial random noise stimulation (tRNS): A pilot study. Frontiers in Psychology, 5(DEC), 1402. https://doi.org/10.3389/fpsyg.2014.01402

Chakravarthi, R., \& Pelli, D. G. (2011). The same binding in contour integration and crowding. Journal of Vision, 11(2011), 1-12. https://doi.org/10.1167/11.8.10

Chen, P. L., Chen, J. T., Fu, J. J., Chien, K. H., \& Lu, D. W. (2008). A pilot study of anisometropic amblyopia improved in adults and children by perceptual learning: An alternative treatment to patching. Ophthalmic and Physiological Optics, 28(5), 422-428. https://doi.org/10.1111/j.1475-1313.2008.00588.x

Chung, S. T. L., Li, R. W., \& Levi, D. M. (2006). Identification of contrast-defined letters benefits 
from perceptual learning in adults with amblyopia. Vision Research, 46(22), 3853-3861. https://doi.org/10.1016/j.visres.2006.06.014

Chung, S. T. L., Li, R. W., \& Levi, D. M. (2008). Learning to identify near-threshold luminancedefined and contrast-defined letters in observers with amblyopia. Vision Research, 48(27), 2739-2750. https://doi.org/10.1016/j.visres.2008.09.009

Flom, M., Weymouth, F., \& Kahneman, D. (1963). Visual Resolution and Contour Integration. Journal of the Optical Society of America, 53(9), 1026-32. https://doi.org/10.1364/JOSA.53.001026

Gais, S., Plihal, W., Wagner, U., \& Born, J. (2000). Early sleep triggers memory for early visual discrimination skills. Nature Neuroscience, 3(12), 1335-9. https://doi.org/10.1038/81881

Giaschi, D. E., Regab, D., Kraft, S. P., \& Kothe, A. C. (1993). Crowding and Contrast in Amblyopia. Optometry and Vision Science, 70(3), 192-197. https://doi.org/10.1097/00006324199303000-00003

Gilbert, C. D., \& Wiesel, T. N. (1989). Columnar specificity of intrinsic horizontal and corticocortical connections in cat visual cortex. The Journal of Neuroscience: The Official Journal of the Society for Neuroscience, 9(7), 2432-2442. Retrieved from http://www.ncbi.nlm.nih.gov/pubmed/2746337

Grassi, M., \& Soranzo, A. (2009). MLP: a MATLAB toolbox for rapid and reliable auditory threshold estimation. Behavior Research Methods, 41(1), 20-28. https://doi.org/10.3758/BRM.41.1.20

Green, D. M. (1990). Stimulus selection in adaptive psychophysical procedures. J Acoust Soc Am, 87(6), 2662-2674. https://doi.org/10.1121/1.399058

Green, D. M. (1993). A maximum-likelihood method for estimating thresholds in a yes-no task. The Journal of the Acoustical Society of America, 93(4), 2096. https://doi.org/10.1121/1.406696

Huang, C.-B., Zhou, Y., \& Lu, Z.-L. (2008). Broad bandwidth of perceptual learning in the visual system of adults with anisometropic amblyopia. Proceedings of the National Academy of Sciences, 105(10), 4068-73. https://doi.org/10.1073/pnas.0800824105

Hussain, Z., Webb, B. S., Astle, A. T., \& McGraw, P. V. (2012). Perceptual learning reduces crowding in amblyopia and in the normal periphery. The Journal of Neuroscience, 32(2), 474 480. https://doi.org/10.1523/JNEUROSCI.3845-11.2012

Karni, A., \& Sagi, D. (1993). The time course of learning a visual skill. Nature, 365(September), 250-252. https://doi.org/10.1038/365250a0 
Lamme, V. A. F., Supèr, H., \& Spekreijse, H. (1998). Feedforward, horizontal, and feedback processing in the visual cortex. Current Opinion in Neurobiology, 8(4), 529-535. https://doi.org/10.1016/S0959-4388(98)80042-1

Levi, D. M. (2005). Perceptual learning in adults with amblyopia: A reevaluation of critical periods in human vision. Developmental Psychobiology, 46(3), 222-232. https://doi.org/10.1002/dev.20050

Levi, D. M. (2012). Prentice Award Lecture 2011. Optometry and Vision Science, 89(6), 827-838. https://doi.org/10.1097/OPX.0b013e318257a187

Levi, D. M., Hariharan, S., \& Klein, S. A. (2002). Suppressive and facilitatory spatial interactions in amblyopic vision. Vision Research, 42(11), 1379-1394. https://doi.org/10.1016/S00426989(02)00061-5

Levi, D. M., \& Polat, U. (1996). Neural plasticity in adults with amblyopia. Investigative Ophthalmology and Visual Science, 37(3). https://doi.org/10.1073/pnas.93.13.6830

Levi, D. M., Polat, U., \& Hu, Y. S. (1997). Improvement in Vernier acuity in adults with amblyopia: Practice makes better. Investigative Ophthalmology and Visual Science, 38(8), $1493-1510$.

Levitt, H. (1971). Transformed Up-Down Methods in Psychoacoustics. The Journal of the Acoustical Society of America, 49(2), 467-477. https://doi.org/10.1121/1.1912375

Li, J., Thompson, B., Deng, D., Chan, L. Y. L., Yu, M., \& Hess, R. F. (2013). Dichoptic training enables the adult amblyopic brain to learn. Current Biology, 23(8), R308-R309. https://doi.org/10.1016/j.cub.2013.01.059

Li, R. W., Klein, S. A., \& Levi, D. M. (2008). Prolonged perceptual learning of positional acuity in adult amblyopia: perceptual template retuning dynamics. Journal of Neuroscience, 28(52), 14223-14229. https://doi.org/10.1523/JNEUROSCI.4271-08.2008

Li, R. W., \& Levi, D. M. (2004). Characterizing the mechanisms of improvement for position discrimination in adult amblyopia. Journal of Vision, 4(6), 476-87. https://doi.org/10:1167/4.6.7

Li, R. W., Provost, A., \& Levi, D. M. (2007). Extended perceptual learning results in substantial recovery of positional acuity and visual acuity in juvenile amblyopia. Investigative Ophthalmology and Visual Science, 48(11), 5046-5051. https://doi.org/10.1167/iovs.07-0324

Li, R. W., Young, K. G., Hoenig, P., \& Levi, D. M. (2005). Perceptual learning improves visual performance in juvenile amblyopia. Investigative Ophthalmology and Visual Science, 46(9), 3161-3168. https://doi.org/10.1167/iovs.05-0286 
Li, W., Howard, J. D., Parrish, T. B., \& Gottfried, J. A. (2008). Aversive Learning Enhances Perceptual and Cortical Discrimination of Indiscriminable Odor Cues. Science, 319(5871), 1842-1845. https://doi.org/10.1126/science.1152837

Liu, X. Y., Zhang, T., Jia, Y. L., Wang, N. L., \& Yu, C. (2011). The therapeutic impact of perceptual learning on juvenile amblyopia with or without previous patching treatment. Investigative Ophthalmology and Visual Science, 52(3), 1531-1538. https://doi.org/10.1167/iovs.10-6355

Maniglia, M., Pavan, A., Cuturi, L. F., Campana, G., Sato, G., \& Casco, C. (2011). Reducing crowding by weakening inhibitory lateral interactions in the periphery with perceptual learning. PLoS ONE, 6(10), e25568. https://doi.org/10.1371/journal.pone.0025568

Maniglia, M., Pavan, A., Sato, G., Contemori, G., Montemurro, S., Battaglini, L., \& Casco, C. (2016). Perceptual learning leads to long lasting visual improvement in patients with central vision loss. Restorative Neurology and Neuroscience, 34(5), 697-720. https://doi.org/10.3233/RNN-150575

Pediatric Eye Disease Investigator Group. (2003). A randomized trial of prescribed patching regimens for treatment of severe amblyopia in children. Ophthalmology, 110(11), 2075-2087.

Pediatric Eye Disease Investigator Group. (2003). A randomized trial of prescribed patching regimens for treatment of severe amblyopia in children. Ophthalmology, 110(11), 2075-2087.

Pelli, D. G. (1997). The VideoToolbox software for visual psychophysics: transforming numbers into movies. Spatial Vision, 10(4), 437-442. https://doi.org/10.1163/156856897X00366

Polat, U. (2009). Making perceptual learning practical to improve visual functions. Vision Research, 49(21), 2566-2573. https://doi.org/10.1016/j.visres.2009.06.005

Polat, U., Bonneh, Y., Ma-Naim, T., Belkin, M., \& Sagi, D. (2005). Spatial interactions in amblyopia: Effects of stimulus parameters and amblyopia type. Vision Research, 45(11), 1471-1479. https://doi.org/10.1016/j.visres.2004.12.014

Polat, U., Ma-Naim, T., Belkin, M., \& Sagi, D. (2004). Improving vision in adult amblyopia by perceptual learning. Proceedings of the National Academy of Sciences of the United States of America (Vol. 101). https://doi.org/10.1073/pnas.0401200101

Polat, U., Mizobe, K., Pettet, M. W., Kasamatsu, T., \& Norcia, A. M. (1998). Collinear stimuli regulate visual responses depending on cell's contrast threshold. Nature, 391(6667), 580-584. https://doi.org/10.1038/35372

Polat, U., Sagi, D., \& Norcia, A. M. (1997). Abnormal long-range spatial interactions in amblyopia. Vision Research, 37(6), 737-744. https://doi.org/10.1016/S0042-6989(96)00154-X 
Robol, V., Grassi, M., \& Casco, C. (2013). Contextual influences in texture-segmentation: Distinct effects from elements along the edge and in the texture-region. Vision Research, 88, 1-8. https://doi.org/10.1016/j.visres.2013.05.010

Sagi, D. (2011). Perceptual learning in Vision Research. Vision Research, 51(13), 1552-1566. https://doi.org/10.1016/j.visres.2010.10.019

Simmers, A. J., Gray, L. S., McGraw, P. V., \& Winn, B. (1999). Functional visual loss in amblyopia and the effect of occlusion therapy. Investigative Ophthalmology and Visual Science, 40(12), 2859-2871. https://doi.org/10.1016/S0161-6420(02)01189-2

Stickgold, R., Whidbee, D., Schirmer, B., Patel, V., \& Hobson, J. A. (2000). Visual discrimination task improvement: A multi-step process occurring during sleep. Journal of Cognitive Neuroscience, 12(2), 246-254. https://doi.org/10.1162/089892900562075

To, L., Thompson, B., Blum, J. R., Maehara, G., Hess, R. F., \& Cooperstock, J. R. (2011). A game platform for treatment of amblyopia. IEEE Transactions on Neural Systems and Rehabilitation Engineering, 19(3), 280-289. https://doi.org/10.1109/TNSRE.2011.2115255

Walker, M. P., Stickgold, R., Jolesz, F. A., \& Yoo, S. S. (2005). The functional anatomy of sleepdependent visual skill learning. Cerebral Cortex, 15(11), 1666-1675. https://doi.org/10.1093/cercor/bhi043

Wong, E. H., Levi, D. M., \& McGraw, P. V. (2005). Spatial interactions reveal inhibitory cortical networks in human amblyopia. Vision Research, 45(21), 2810-2819. https://doi.org/10.1016/j.visres.2005.06.008

Zhou, Y., Huang, C., Xu, P., Tao, L., Qiu, Z., Li, X., \& Lu, Z. L. (2006). Perceptual learning improves contrast sensitivity and visual acuity in adults with anisometropic amblyopia. Vision Research, 46(5), 739-750. https://doi.org/10.1016/j.visres.2005.07.031 
Table 1

\begin{tabular}{|c|c|c|c|c|c|c|c|c|c|c|}
\hline $\begin{array}{l}\text { Partici- } \\
\text { pants }\end{array}$ & $\begin{array}{l}\text { Gen- } \\
\text { der }\end{array}$ & Age & $\begin{array}{l}\text { Refractive } \\
\text { error }\end{array}$ & $\begin{array}{l}\text { Visual } \\
\text { acuity } \\
\text { logMAR }\end{array}$ & $\begin{array}{l}\text { Stra- } \\
\text { bism }\end{array}$ & $\begin{array}{l}\text { Suppres- } \\
\text { sion }\end{array}$ & $\begin{array}{l}\text { Stereopsi } \\
\mathbf{s}\end{array}$ & $\begin{array}{l}\text { Eye } \\
\text { alignment }\end{array}$ & $\begin{array}{l}\text { Cause of } \\
\text { Amblyopia }\end{array}$ & $\begin{array}{l}\text { Occlusion } \\
\text { therapy }\end{array}$ \\
\hline P1 & $F$ & 10,8 & $\begin{array}{l}\text { RE } \\
+10.00 \\
+2.00 \times 170 \\
\text { LE } \\
\text { plano }\end{array}$ & $\frac{\text { RE .78, }}{\text { LE }-.02}$ & No & No & Absent & Ortho & $\begin{array}{l}\text { Aniso- } \\
\text { metropia }\end{array}$ & No \\
\hline P2 & $F$ & 9,9 & $\begin{array}{l}\mathrm{RE} \\
+6.75-1.50 \times 180 \\
\mathrm{LE} \\
+6.75-1.75 \times 160\end{array}$ & $\begin{array}{l}\text { RE .06, } \\
\text { LE . } 18\end{array}$ & Yes & No & $\begin{array}{l}\text { 70" with } \\
\text { Randot }\end{array}$ & Exotropia & Strabismus & failed \\
\hline P3 & $\mathrm{F}$ & 7,8 & $\begin{array}{l}\text { RE } \\
+2.00 \\
\text { LE } \\
+2.00\end{array}$ & $\begin{array}{l}\text { RE .05, } \\
\text { LE .42 }\end{array}$ & Yes & LE & Not tested & Exotropia & Strabismus & interrupted \\
\hline P4 & M & 25,3 & $\begin{array}{l}\text { RE } \\
+0.50-4.50 \times 140 \\
\text { LE } \\
+2.00-4.75 \times 30 \\
\end{array}$ & $\begin{array}{l}\text { RE .18, } \\
\text { LE .42 }\end{array}$ & Yes & LE & Coarse & Exotropia & Strabismus & irregualar \\
\hline P5 & M & 11,7 & $\begin{array}{l}\mathrm{RE} \\
+4.25+1.50 \times 135 \\
\mathrm{LE} \\
+4.25+2.50 \times 94\end{array}$ & $\begin{array}{l}\text { RE .02, } \\
\text { LE . } 73 \\
\end{array}$ & Yes & LE & Absent & Esotropia & Strabismus & irregular \\
\hline P6 & M & 7,3 & $\begin{array}{l}\mathrm{RE} \\
+3.00+1.25 \times 85 \\
\mathrm{LE} \\
+7.75+0.75 \times 88\end{array}$ & $\begin{array}{l}\text { RE }-.15, \\
\text { LE } .23 \\
\end{array}$ & Yes & LE & Absent & Exotropia & $\begin{array}{l}\text { Strabismus } \\
\& \text { aniso- } \\
\text { metropia }\end{array}$ & $\begin{array}{l}\text { irregualar } \\
\text { delated }\end{array}$ \\
\hline P7 & $M$ & 7,5 & $\begin{array}{l}\text { RE } \\
-8.25-2.50 \times 150 \\
\text { LE } \\
\text { plano } \\
\end{array}$ & $\frac{\text { RE } .88,}{\text { LE }-.12}$ & No & No & $240 "$ & Ortho & $\begin{array}{l}\text { Aniso- } \\
\text { metropia }\end{array}$ & $\begin{array}{l}\text { irregualar } \\
\text { delated }\end{array}$ \\
\hline P8 & $F$ & 47,2 & $\begin{array}{l}\text { RE } \\
\text { plano } \\
\text { LE } \\
-0.25+1.00 \times 80 \\
\end{array}$ & $\frac{\text { RE . } 37}{\text { LE .009 }}$ & No & No & Unknown & Ortho & Unclassified & No \\
\hline P9 & $M$ & 21,8 & $\begin{array}{l}\mathrm{RE} \\
+4.50+2.50 \times 110 \\
\mathrm{LE} \\
+5.00+2.50 \times 70\end{array}$ & $\frac{\text { RE } .63}{\text { LE } .40}$ & No & No & Unknown & $\begin{array}{l}\text { Nystag- } \\
\text { mus }\end{array}$ & $\begin{array}{l}\text { Bilateral } \\
\text { ametropia }\end{array}$ & No \\
\hline P10 & $M$ & 53,0 & $\begin{array}{l}\mathrm{RE} \\
+0.50-1.00 \times 140 \\
\mathrm{LE} \\
+0.25-0.75 \times 25 \\
\end{array}$ & $\begin{array}{l}\text { RE }-.07, \\
\text { LE } .6 \\
\end{array}$ & Yes & No & No & Esotropia & Strabismus & $\begin{array}{l}\text { irregular } \\
\text { interrupted }\end{array}$ \\
\hline \multicolumn{2}{|c|}{$\begin{array}{c}\text { Controls } \\
\text { (M+F) } \\
\text { n.10 }\end{array}$} & $\begin{array}{c}7-51 \\
( \pm 16.9)\end{array}$ & & & No & No & Yes & Regular & No & No \\
\hline
\end{tabular}

Table 1. Clinical details of each amblyopic observer. Visual acuity is expressed in logMAR (MAR, minimum angle of resolution in arc min); LE, left eye (in bold and underlined if is the amblyopic eye); RE, right eye (in bold and underlined if is the amblyopic eye). 
Table 2

\begin{tabular}{|c|c|c|c|c|c|c|}
\hline $\begin{array}{l}\text { Partici- } \\
\text { pant }\end{array}$ & $\begin{array}{l}\text { SF } \\
\left(\text { cycles } /^{\circ}\right)\end{array}$ & $\begin{array}{l}\text { Stimulus } \\
\text { duration (ms) }\end{array}$ & $\begin{array}{l}\text { N. sess. } \\
\text { per SF }\end{array}$ & $\begin{array}{l}\text { Tot. } \\
\text { n. sess. }\end{array}$ & $\begin{array}{l}\text { PL duration } \\
\text { per SF } \\
\text { (month) }\end{array}$ & $\begin{array}{l}\text { Tot. PL } \\
\text { duration } \\
\text { (months) }\end{array}$ \\
\hline P1 & $3,3,6$ & $500,250,250$ & $18,9,27$ & 54 & $1.4,0.5,2.1$ & 4 \\
\hline P2 & $3,11,9,11$ & $133,133,133,133$ & $8,20,25,19$ & 72 & $1.2,3.4,2.3,3.8$ & 10.7 \\
\hline P3 & 3,8 & 133,133 & 28,8 & 36 & $3.0,0.9$ & 3.9 \\
\hline P4 & 3,6 & 250,250 & 33,31 & 64 & $4.2,1.4$ & 5.6 \\
\hline P5 & $3,3,6,6,8$ & $\begin{array}{l}133,250,133,250 \\
250\end{array}$ & $22,11,19,19,18$ & 89 & $\begin{array}{l}1.3,0.6,1.2,1.3, \\
1.1\end{array}$ & 5.5 \\
\hline P6 & $3,5,6,6$ & $133,133,133,133$ & $27,30,22,14$ & 93 & $1.2,1.5,1.3,4.1$ & 8.1 \\
\hline P7 & 3,4 & 133,133 & 24,13 & 37 & $2.7,2.2$ & 4.9 \\
\hline P8 & 6 & 133 & 21 & 21 & 4,2 & 4.2 \\
\hline P9 & $3,3,4,6$ & $250,133,133,133$ & $7,4,12,20$ & 43 & $0.5,0.3,1.2,2.1$ & 4.1 \\
\hline P10 & $10,11,12,15$ & $200,200,200,200$ & $9,12,16,46$ & 83 & $0.5,1,2.3,3.1$ & 6.9 \\
\hline
\end{tabular}

Table 2. The spatial frequencies (SF) and stimulus duration used during training. Details of training duration and number of training session are also reported. 
Table 3

\begin{tabular}{|c|c|c|c|c|c|c|c|c|c|c|c|}
\hline \multirow{2}{*}{$\begin{array}{l}\text { Partici- } \\
\text { pants }\end{array}$} & \multirow[t]{2}{*}{ Date } & \multicolumn{2}{|c|}{ VA (logMAR) } & \multicolumn{6}{|c|}{ CONTRAST SENSITIVITY $\left(\right.$ cycles $/^{\circ}$ ) } & \multirow{2}{*}{$\frac{\text { Vernier }}{\text { arcsec }}$} & \multirow{2}{*}{$\frac{\text { CW }}{\text { Gap c-c }}$} \\
\hline & & F Eye & A Eye & 1 & 3 & 5 & $\overline{7}$ & 11 & 15 & & \\
\hline \multirow[t]{2}{*}{$\mathbf{P 1}$} & $03 / 06 / 2013$ & -0.02 & 0.78 & 18.76 & 7.531 & 2.44 & 0.00 & 0.00 & & & 11.5 \\
\hline & $07 / 10 / 2013$ & & 0.86 & 27.17 & 17.75 & 5.69 & 3.57 & 7.82 & & & 6.9 \\
\hline$f$-up & $18 / 01 / 2016$ & -0.27 & 0.82 & 31.45 & 18.69 & 5.00 & 2.57 & & & & 7.7 \\
\hline \multirow[t]{2}{*}{$\overline{\mathbf{P 2}}$} & $25 / 07 / 2013$ & 0.06 & 0.18 & 39.68 & 91.74 & 30.86 & 20.37 & 13.26 & 3.13 & 249.2 & 6.00 \\
\hline & $03 / 12 / 2014$ & -0.30 & -0.23 & 44.64 & 109.8 & 222.2 & 103 & 106.3 & 91.74 & 62.4 & 5.0 \\
\hline \multirow[t]{2}{*}{ P3 } & $18 / 02 / 2014$ & -0.05 & 0.42 & 153.8 & 104.1 & 28.98 & 14.4 & 7.86 & 2.56 & & \\
\hline & $13 / 06 / 2014$ & -0.08 & 0.29 & 86.95 & 222.2 & 30.39 & 25.9 & 13.07 & 5.065 & & \\
\hline$f$-up & $07 / 12 / 2015$ & & 0.03 & 153.0 & 336 & 33 & 36 & 19 & 7 & & \\
\hline \multirow[t]{2}{*}{$\mathbf{P 4}$} & $19 / 06 / 2014$ & 0.18 & 0.52 & 135.1 & 192.3 & 39.84 & 88.50 & 16.05 & 3.21 & & 19.6 \\
\hline & 19/02/2015 & -0.02 & 0.13 & & 135.1 & 85.47 & 86.95 & 19.8 & 4.99 & & 6.9 \\
\hline$f$-up & $29 / 09 / 2015$ & 0.14 & 0.13 & 86.95 & 156.2 & 204.0 & 147.0 & 31.74 & & & 7.7 \\
\hline \multirow[t]{2}{*}{$\overline{\mathrm{P} 5}$} & $26 / 06 / 2014$ & 0.02 & 0.73 & 46.30 & 21.28 & 8.46 & 4.81 & & & 131.6 & 7.1 \\
\hline & $22 / 04 / 2015$ & & 0.55 & 120.5 & 51.81 & 17.42 & 19.53 & 7.42 & & 69.1 & 5.0 \\
\hline$f$-up & $21 / 10 / 2015$ & -0.15 & 0.31 & 33.67 & 74.63 & 52.36 & 37.04 & 7.60 & & 50.4 & \\
\hline \multirow[t]{2}{*}{$\overline{P 6}$} & $01 / 09 / 2014$ & -0.15 & 0.23 & 93.46 & 39.22 & 37.59 & 14.60 & 5.44 & 1.89 & & 7.8 \\
\hline & $16 / 06 / 2015$ & & 0.18 & 54.95 & 80.00 & 24.21 & 30.40 & 9.80 & & 93.7 & \\
\hline$f$-up & $29 / 09 / 2015$ & -0.06 & 0.05 & 131.5 & 131.5 & 158.7 & 79.37 & 31.45 & & 23.3 & \\
\hline \multirow[t]{2}{*}{$\overline{\mathbf{P 7}}$} & $30 / 04 / 2014$ & -0.12 & 0.88 & 434.7 & 86.96 & 16.05 & 6.61 & & & 281 & 5.0 \\
\hline & $15 / 05 / 2015$ & & 0.72 & 163.9 & 39.06 & 14.60 & 6.52 & & & 277 & \\
\hline f-up & $15-12-2015$ & & 0.62 & & & & & & & & \\
\hline \multirow[t]{2}{*}{ P8 } & $25 / 09 / 2014$ & 0.01 & 0.37 & 29.15 & 43.80 & 18.72 & 18.51 & 4.08 & 2.44 & 250.7 & 13.3 \\
\hline & $08 / 04 / 2015$ & -0.01 & 0.17 & 50.50 & 40.48 & 20.28 & 25.00 & 6.27 & 3.47 & 250.7 & 9.8 \\
\hline \multirow[t]{2}{*}{$\overline{\mathrm{P9}}$} & $15 / 09 / 2015$ & & 0.63 & 107.5 & 38.57 & 5.34 & 0 & & & 60.8 & 5.0 \\
\hline & 28/01/2016 & & 0.40 & 72 & 69 & 5 & 1.68 & & & 48 & 5.0 \\
\hline$f$-up & & & 0.42 & 1412 & 24 & 8 & 5 & & & 33 & 7.7 \\
\hline \multirow[t]{3}{*}{$\mathrm{P} 10$} & $08 / 02 / 2016$ & -0.07 & 0.6 & 78.13 & 98.04 & 69.44 & 78.74 & 18.98 & 8.29 & 95 & 21.6 \\
\hline & 05/05/2016 & -0.14 & 0.5 & 67.67 & 90.91 & 133.3 & 92.59 & 20 & 7.7 & 64 & 9,9 \\
\hline & $19 / 12 / 2016$ & & 0.46 & & & & & & & & \\
\hline $\begin{array}{l}\text { Control } \\
\text { group } \\
(\mathrm{N}=10)\end{array}$ & $\begin{array}{c}\text { Test } \\
\text { Retest }\end{array}$ & $\begin{array}{l}-0.15 \\
-0.15\end{array}$ & & $\begin{array}{l}88.46 \\
83.75\end{array}$ & $\begin{array}{l}150.4 \\
128.2\end{array}$ & $\begin{array}{l}114.3 \\
114.3\end{array}$ & $\begin{array}{l}68.11 \\
74.25\end{array}$ & $\begin{array}{l}42.93 \\
48.35\end{array}$ & $\begin{array}{l}30.51 \\
29.03\end{array}$ & $\begin{array}{l}4.89 \\
5.62\end{array}$ & $\begin{array}{l}0.20 \\
0.24\end{array}$ \\
\hline
\end{tabular}

Table 3. Transfer data for the amblyopic eye measured before PL, after, and at follow-up (f-up): SLOAN acuity, CSF for spatial frequencies of $1,3,5,7,11$, and 15 cycles/ ${ }^{\circ}$, Vernier acuity, and foveal crowding expressed as center-to-center critical spacing in stroke units (Gap c-c). SLOAN data were also measured for the fellow eye. Data from the control group refers to the Test-Retest. 


\section{Figure Captions}

Figure 1. Representation of the stimuli used in the training phase and in pre- and post-training to test for lateral interactions. Only the vertical global configuration is shown, from left to right $2 \lambda, 3 \lambda$, $4 \lambda$, and $8 \lambda$ are shown. See text for more details about the stimuli.

Figure 2. Top panels (A and B) show contrast thresholds as a function of the target-to-flankers separation obtained before and after PL to low (A) and high spatial frequencies (B). Bottom panels show threshold modulation as a function of the target-to-flankers separation, resulting from PL to low (C) and high spatial frequencies (D). Zero indicates no modulation, whereas negative values indicate reduced suppression. Error bars \pm SEM.

Figure 3. Individual and average visual acuity measured before and after PL and at the follow-up in the amblyopic eye (A) and fellow eye (B). Error bars \pm SEM.

Figure 4. (A) Normalized contrast sensitivity function estimated before training (empty circles and dotted line), after training (filled black circles and continuous black line), and at follow-up (grey circles and continuous gray line). (B) PL-dependent enhancement in contrast sensitivity expressed as the log differences between contrast sensitivity before and after the training (empty squares and dotted line) and between contrast sensitivity estimated after training and at the follow-up (filled black squares and continuous line). Error bars \pm SEM.

Figure 5. Individual and mean Vernier acuity measured in the amblyopic eye before and after PL. Error bars \pm SEM.

Figure 6. Individual and mean critical spacing measured in the amblyopic eye before and after PL. The value reported compares center-center critical spacing expressed in stroke-units. Error bars \pm SEM. 
Figure 1

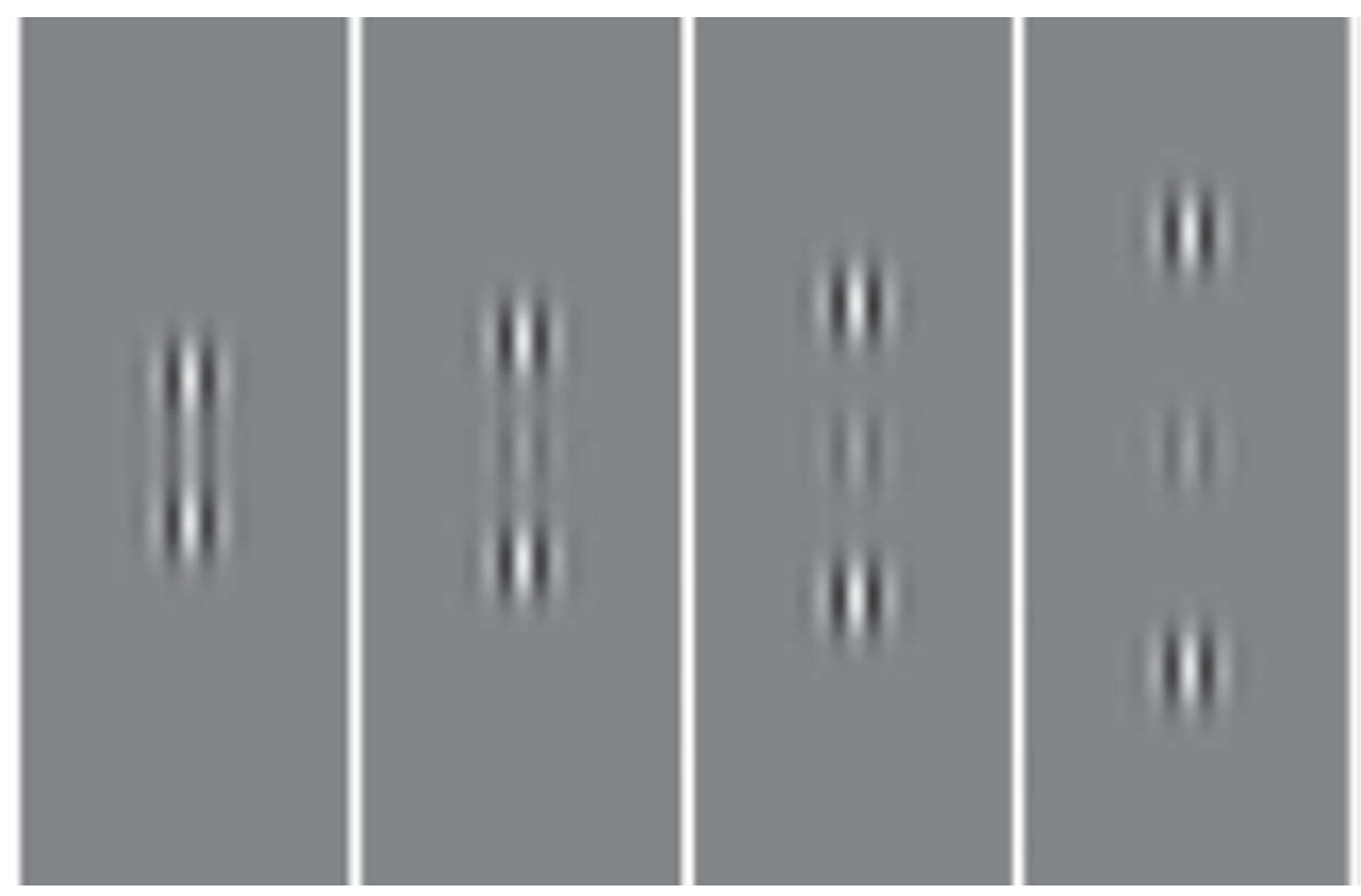


Figure 2

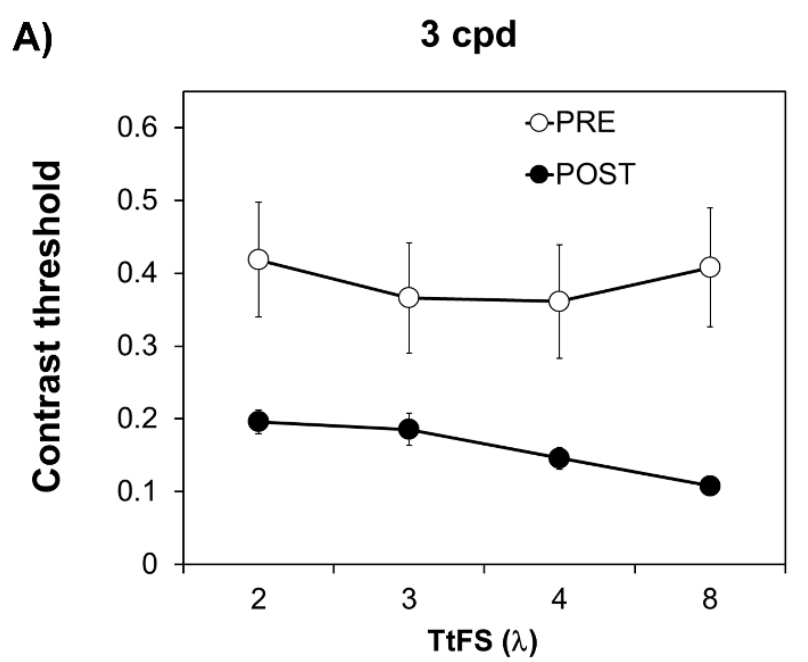

B) $\quad \geq 6 \mathrm{cpd}$

C)
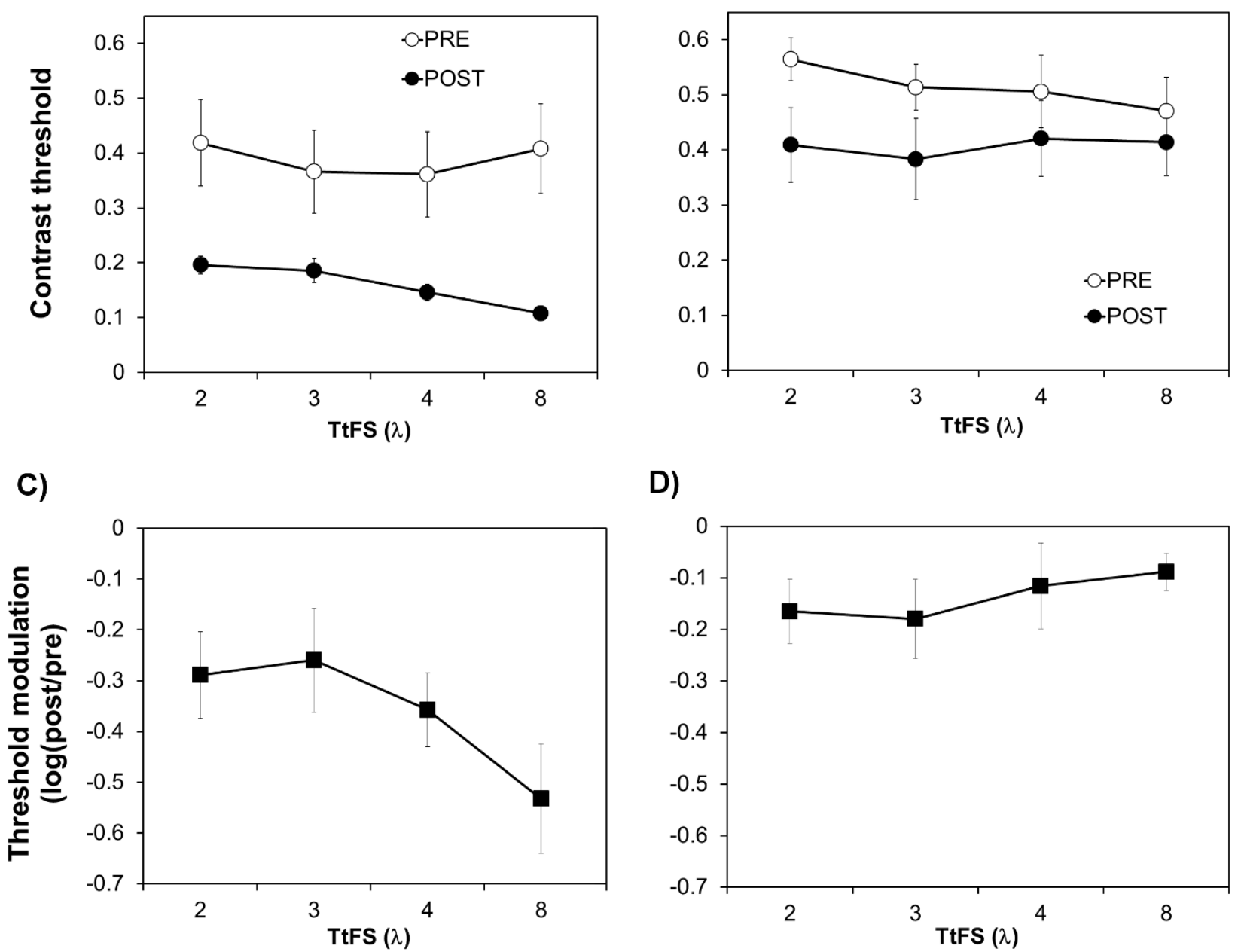

D)

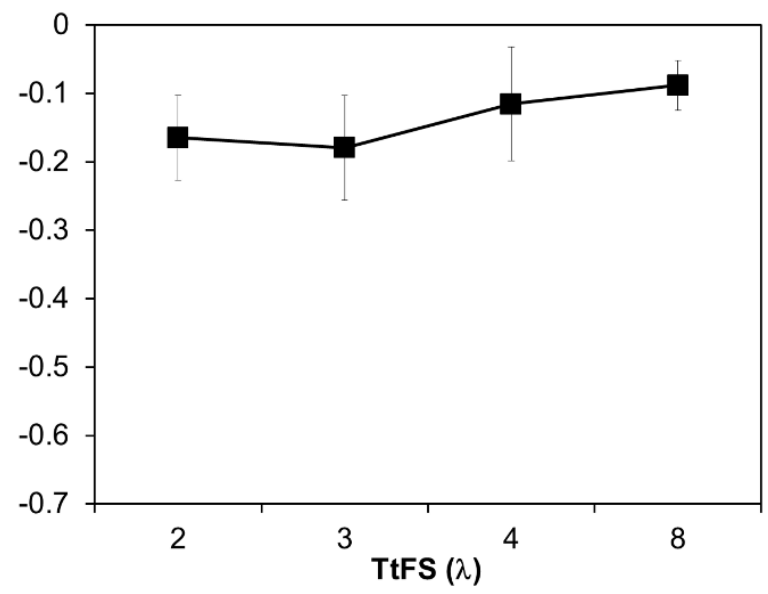


Figure 3

A)

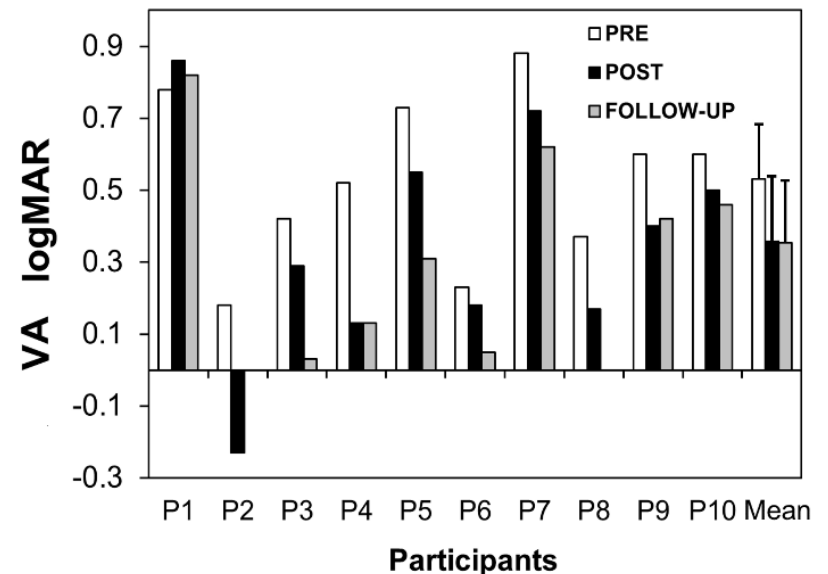

B)

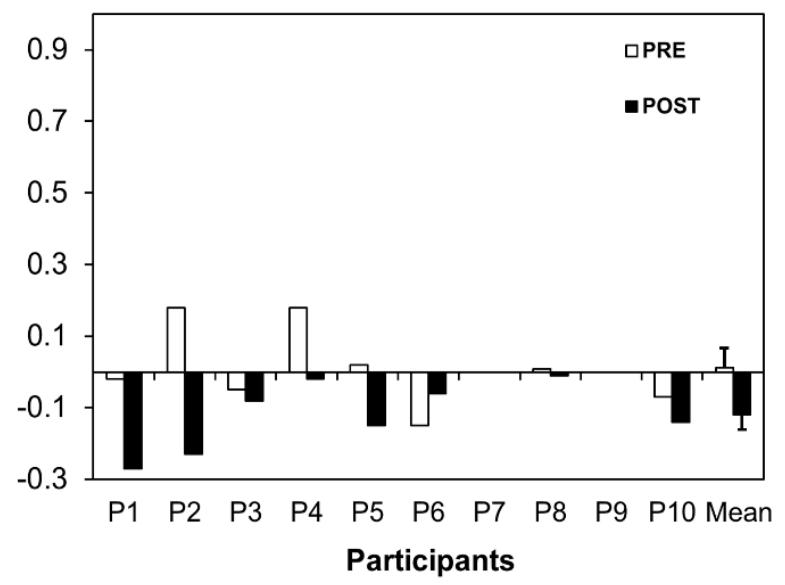


Figure 4

A)

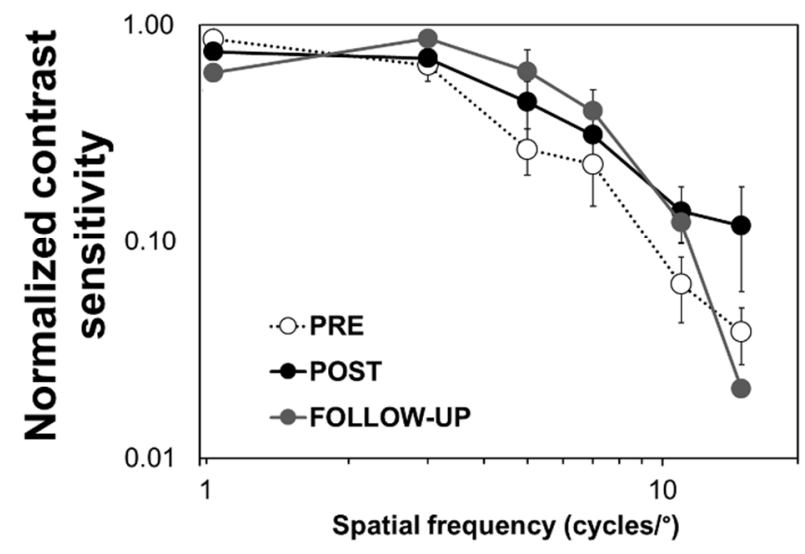

B)

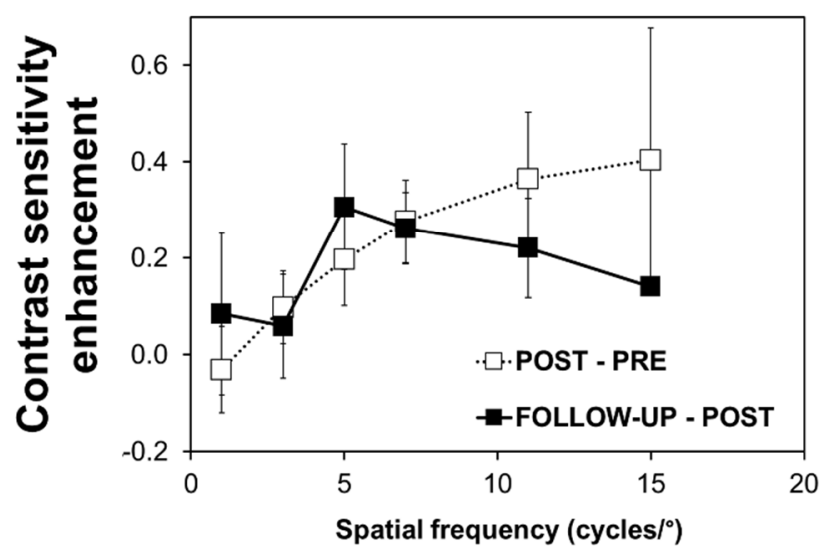


Figure 5

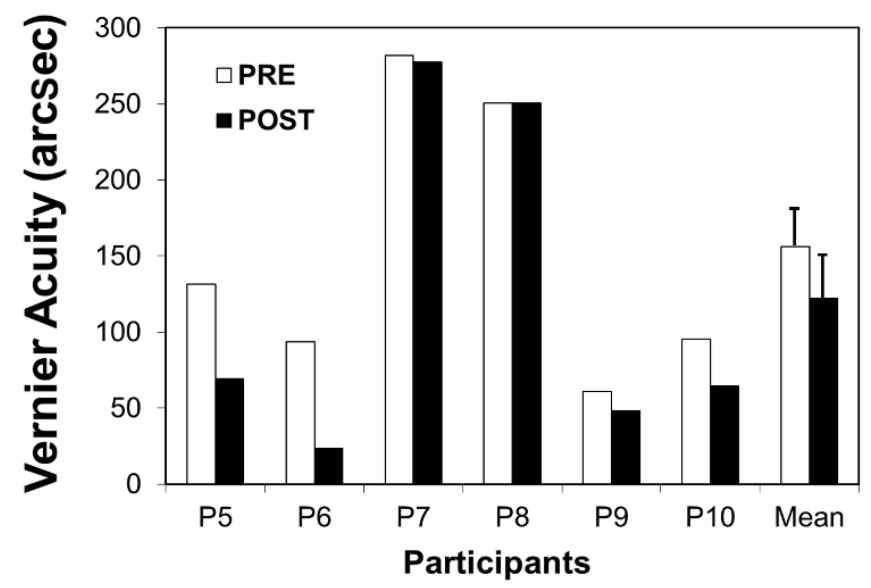


Figure 6

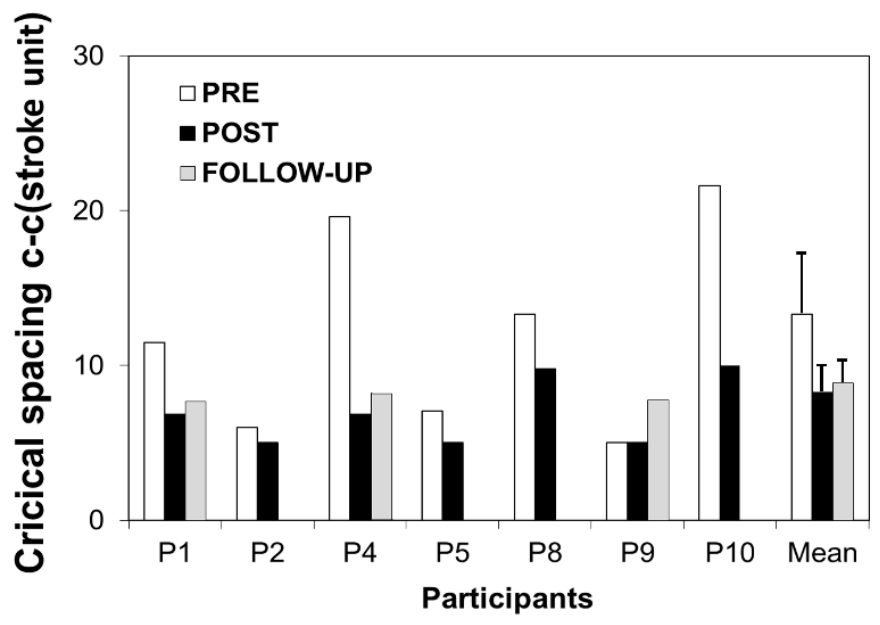

